\title{
Moderating the route angularity effect in a virtual environment: Support for a dual memory representation
}

\author{
Adam T. HutCheson ANd Douglas H. Wedell \\ University of South Carolina, Columbia, South Carolina
}

\begin{abstract}
Research has shown that increasing the number of turns that a route takes through the environment increases estimates of distance - the route angularity effect. This study tested implications of different memory-based explanations of the route angularity effect within a virtual setting. Participants maneuvered through virtual pathways of varying length that included zero, two, or seven turns. After each set of three paths, they estimated relative path lengths on an analog scale. Results demonstrated that both increasing memory load during navigation and making retrieval more difficult by interpolating another spatial task prior to estimation significantly increased the magnitude of route angularity effects. These results are consistent with the idea that the number of turns is categorically encoded and used as a memory heuristic when fine-grained memory for the route distance is degraded either at encoding or prior to retrieval.
\end{abstract}

Many factors can lead to biased estimates of distance. For example, increasing the number of environmental features encountered on a path, the amount of time it takes to travel a path, or the degree of effort expended in traveling a path all lead to a positive bias in distance estimates (Allen, 1981; Allen \& Kirasic, 1985; Montello, 1997). The biasing environmental feature that we focus on in the research presented here is the number of turns a route takes. Sadalla and Magel (1980) demonstrated that the more turns there are on a path, the greater the estimate of its distancereferred to as the route angularity effect. In their studies, participants walked along paths that were marked on hallway floors and that contained multiple right-angle turns. The paths were traversed in pairs and included either two or seven turns. Participants were asked to draw maps of the paths to give their distance estimates or, alternatively, to reproduce the length of a path by walking that distance in a straight line. Their findings supported previous research conducted in natural settings that suggested increases in the number of turns lead to increases in the estimated distance, independent of any effects of travel time (Briggs, 1973; Lee, 1973; Stea, 1969).

Although the route angularity effect is well established, the mechanisms underlying it have yet to be fully determined. The principal and most compelling explanations of the route angularity effect have linked the phenomenon to memory processes (Jansen-Osmann \& Wiedenbauer, 2004, 2006; Montello, 1997; Sadalla \& Magel, 1980), but these explanations have typically not been tested directly. Indeed, different memory explanations of the route angularity effect appear to make different predictions about the consequences of disrupting encoding of information during route navigation. We tested these explanations in the present study by manipulating the task structure to examine how disruptions at encoding or prior to retrieval impact the magnitude of the route angularity effect. Thus, we used distance estimation in a virtual environment to examine how manipulations related to memory encoding and retrieval directly impact the magnitude of the route angularity effect. Before proceeding to experimental details, we first describe memory explanations of the route angularity effect and the occurrence of the effect in virtual environments.

\section{Memory Explanations of the \\ Route Angularity Effect}

Several researchers have argued that the route angularity effect arises out of memory features associated with the task (Jansen-Osmann \& Berendt, 2002; JansenOsmann \& Wiedenbauer, 2004, 2006; Sadalla \& Magel, 1980; Sadalla, Staplin, \& Burroughs, 1979). As Montello (1997) pointed out, the route angularity effect can be considered one of several related phenomena captured by a more general feature accumulation hypothesis. According to this hypothesis, as one navigates through an environment, one accumulates memories for features, with distance estimates positively related to the number of features accumulated. If we consider turns as salient features, remembering seven turns along a path should lead to an overestimation of distance relative to remembering only two turns along a path.

In support of the critical role of memory, Sadalla et al. (1979) labeled intersections with high-frequency names or low-frequency names and demonstrated that

A. T. Hutcheson, hutchesa@mailbox.sc.edu 
distance estimation was greater for the path with highfrequency names. Several pieces of evidence supported the critical role of retrieval in mediating this effect. First, high-frequency names were easier to recall but had no advantage in recognition, suggestive of a retrieval component. Second, when participants were prompted with intersection names, the difference in distance estimation disappeared. Finally, prompts that increased information recall also increased distance estimations. Sadalla et al. then demonstrated that factors that make it easier to retrieve more features about a path will increase the estimated distance of the path.

Although Sadalla and colleagues (Sadalla et al., 1979; Staplin \& Sadalla, 1981) provided convincing evidence that implicated retrieval as a key mediator of route angularity and related effects, their approach did not adequately explain why the route angularity effect is sometimes not obtained (Jansen-Osmann \& Wiedenbauer, 2004, 2006). Heft (1988) argued that, in real-world environments, the route angularity effect may not occur, because the turns can be viewed by the observer as not being actual changes. The foundation of his argument was that a change in path direction does not really constitute route angularity when you consider the view of the observer. If the path is very short but contains many turns, the participant can likely see in a straight line through a few of the turns. This will make a path with eight turns seem more like a path with fewer turns, because the participant's viewpoint is not really changing as a result of the environment. He, instead, proposed that the effect is found when the observer experiences a new place or vista. Jansen-Osmann and Wiedenbauer (2006) proposed that task difficulty is the key moderating factor. In particular, they build on the idea of dual codes for spatial memory (Huttenlocher, Hedges, \& Duncan, 1991) to suggest that features, such as the number of turns, only moderate the route angularity effect when analog or fine-grained memory for the route distances is disrupted. They provided indirect support for this relationship by manipulating the number of paths (three or five) that had to be held in memory before making distance estimations, and they found that significant route angularity effects only occurred in the high memory load (five-path) condition.

At first blush, the feature retrieval research reported by Sadalla et al. (1979) and the task demands research reported by Jansen-Osmann and Wiedenbauer (2006) appear to be at odds. This is because the earlier research showed that improving memory for features along a path increased distance estimates, whereas the latter research showed that making it more difficult to remember paths leads to an increase in the route angularity effect. According to the feature accumulation hypothesis, increasing task difficulty should make it harder to retrieve path information and therefore reduce the route angularity effect. This contradiction can be resolved by considering two distinct memory codes for spatial information: categorical and fine-grained memory (Huttenlocher et al., 1991). Categorical information is discrete and can be used to supplement fine-grained information when it is disrupted. Research that has manipulated delays before the estimation of spatial locations has demonstrated that fine-grained memory is fragile and quickly decays, resulting in greater reliance on categorical coding (Fitting, Wedell, \& Allen, 2008a, 2008b; Merchant, Fortes, \& Georgopoulos, 2004; Spencer \& Hund, 2002). Therefore, the resolution to this seeming contradiction is that feature accumulation utilizes categorical codes, which are distinct from the fine-grained codes of spatial distance. When memory demands are low, fine-grained coding is robust, and so categorical coding is not used, resulting in the elimination of the route angularity effect. On the other hand, task features that disrupt fine-grained memory should result in greater reliance on categorical memory and therefore augment the route angularity effect. Although Jansen-Osmann and Wiedenbauer (2006) tested this implication indirectly by manipulating the number of paths held in memory before recall; we tested it more directly by manipulating activities during encoding and prior to retrieval that should increase reliance on categorical coding at retrieval. Before describing these manipulations, we first discuss our use of a virtual environment to test for moderators of the route angularity effect.

\section{The Route Angularity Effect in Virtual Environments}

Virtual environments (VEs) have been an important tool for spatial cognition researchers for many years, because of the rigorous control the experimenter can exercise over environmental variables. Jansen-Osmann and colleagues (Jansen-Osmann \& Berendt, 2002; Jansen-Osmann \& Wiedenbauer, 2004, 2006) pioneered the investigation of the route angularity effect in simulated VEs. Their results demonstrated that, although the route angularity effect can be found in VEs, it is not guaranteed to occur. In an early study, Jansen-Osmann and Berendt focused on how the number of turns found in a VE corridor affects estimates of its distance. In their Experiment 1, a VE displayed on a desktop computer featured corridors with either two turns (Route A), seven turns (Route B), or no turns (Route C). After traversing the routes, the participants were asked to compare the lengths of Route A and Route B (which were the same length) with that of Route $C$, which was shorter, by drawing them. This method allows the two test routes (A and $\mathrm{B}$ ) to be compared with a standard scale of distance (C) against which the participants made their estimates. The participants did not have to make a numeric estimate of path length for the two test paths using this method. They simply estimated the length of the test route by drawing a line that corresponded to the ratio of the target path length to the comparison path length. After comparative judgment, they were asked to draw maps of Route A and Route B as a representation of their distance estimation. Both measures demonstrated that the two-turn Route A was estimated as being shorter than the seven-turn Route $\mathrm{B}\left(\eta^{2}=.31\right.$ for comparative estimation). Jansen-Osmann and Berendt replicated this effect in their Experiment 2 using a much longer comparison route, with the lengths of Routes A and B remaining the same.

Although this initial exploration of route angularity in a VE appeared to set the effect on solid ground, another set of studies (Jansen-Osmann \& Wiedenbauer, 2004) 
called into question the robustness of the effect. In JansenOsmann and Wiedenbauer's (2004) Experiment 1, schoolage children participated in a replication of Experiment 1 of Jansen-Osmann and Berendt (2002). The result demonstrated a route angularity effect that was dependent on the order of presentation. The seven-turn route led to greater distance estimates only when this route was explored after the two-turn route. In their Experiment 2 (using children) and Experiment 3 (using adults), Jansen-Osmann and Wiedenbauer (2004) eliminated the within-subjects manipulation of the number of turns. Rather than exploring both $\mathrm{A}$ and $\mathrm{B}$, the participants explored one corridor and then explored a virtual room filled with objects for the same amount of time that passed in Experiment 1 before moving on to Route C. Neither experiment found a route angularity effect. Jansen-Osmann and Wiedenbauer's (2004) conclusion was that the route angularity effect was dependent on the experimental design, and they offered two explanations of why the within-subjects design would show the route angularity effect but the between-subjects design would not. First, they argued that the comparison of routes in the within-subjects design may result in a relative judgment process, whereas the lack of such comparisons in the between-subjects design forces the participant to make an absolute judgment. Second, they argued that the within-subjects design required participants to maintain more information in memory, which may have led them to rely on a turn-based heuristic in order to estimate distance.

In a follow-up study, Jansen-Osmann and Wiedenbauer (2006) tested how the number of paths that were needed to be held in memory impacted the occurrence of the route angularity effect by manipulating memory load during encoding. Their Experiment 1 was essentially a replication of the three-path procedure used earlier, with the number of routes manipulated within subjects. Unlike the results of previous studies, no main effect of the number of turns and no order effects were found. In their second experiment, they added two additional routes (a two-turn and a seven-turn route), so that the participants explored five routes before making their estimates. They found a significant route angularity effect in the five-path condition $\left(\eta^{2}=.23\right.$ for comparative estimation) and, once again, no order effects. They argued that this pattern of results supported the idea that memory load is the crucial factor that accounts for the route angularity effect: Only when fine-grained or analog memory for the route is degraded will one need to rely on the categorical coding of the number of turns.

\section{Memory Manipulations}

Our experiment was also implemented within a VE and was designed to more carefully explore how memory factors moderate the route angularity effect. The use of the VE enabled us to efficiently manipulate the additional tasks conducted while the participants navigated the route or after navigation but prior to retrieval. In particular, we examined the role of memory in determining the route angularity effect by degrading memory in two ways. First, we manipulated whether the participant had to engage in a spatially demanding concurrent task while navigating the virtual route. This was manipulated within subjects, so that, on half the trials, the participants pressed arrow keys indicating the directions that they were hearing as they navigated the routes, and, on half of the trials, they simply navigated the routes. Second, we manipulated whether a 60 -sec delay between completing the routes and estimating them was filled with a task that demanded spatial resources or was unfilled. This was manipulated between subjects, with those in the filled condition completing a series of mental rotation trials during the delay (Shepard \& Metzler, 1971).

Thus, we had two primary goals. The first was to more firmly establish the link between the route angularity effect and memory. By manipulating memory difficulty in two ways, we could assess whether the route angularity effect was enhanced or diminished when memory was degraded. Earlier memory research based on the accumulation hypothesis implied that degrading memory leads to reduced route angularity effects (Sadalla et al., 1979; Staplin \& Sadalla, 1981). However, a framework based on the dual encoding of fine-grained and categorical information makes the opposite prediction. Since fine-grained memory is fragile and categorical memory is robust, disruption of spatial memory encoding during navigation should result in a greater reliance on categorical codes and should thereby enhance the route-angularity effect. The second goal was to determine whether the effect was dependent on memory processing being degraded during navigation or whether it was a more general phenomenon that extended to disruption of memory after the routes had been traversed. If the route angularity effect is the result of using the number of turns as a heuristic in estimating distance when fine-grained memory is degraded, one would expect the effect to be moderated by both a concurrent task and the type of delay.

In summary, this experiment built on previous studies of the route angularity effect in a VE (Jansen-Osmann \& Berendt, 2002; Jansen-Osmann \& Wiedenbauer, 2006) but added several factors. As in previous studies, we had participants navigate two test paths followed by a comparison path and then had them compare each test path with the comparison path in the set. The comparison path was always the same length $(20 \mathrm{ft})$ and had no turns. The test paths had either two or seven turns and varied in length (40, 80 , or $120 \mathrm{ft}$ ). Unlike in previous work, our participants experienced many sets of paths and made estimates after each set. This provided a more reliable basis for evaluating distance estimates and allowed us to manipulate path length and concurrent task within subjects. Our focus was on two interaction effects predicted by the dual memory encoding model (Huttenlocher et al., 1991). First, we predicted an interaction of the number of turns and the presence of the concurrent task, such that the route angularity effect would be greater when participants were engaged in a concurrent spatial task. Second, we predicted an interaction of the number of turns and the type of delay, such that the route angularity effect would be greater when participants engaged in a mental rotation task prior to making estimates. Thus, our experiment provided a direct test of implications of the dual memory encoding explanation of the route angularity effect. 


\section{METHOD}

\section{Participants and Design}

Participants were 20 men and 20 women from the University of South Carolina who volunteered in exchange for research credit. The five-way factorial design included the following variables: path size $(40,80$, or $120 \mathrm{ft}$ ), number of turns (two or seven), concurrent direction coding task (present or not), delay type (unfilled or filled with mental rotation judgments), and sex of the participant (male or female). Three variables were manipulated within subjects (path size, number of turns, and concurrent task). The dependent variable was the length of the line that the participant drew for each path with turns relative to the length provided for the no-turn comparison path.

\section{Materials and Apparatus}

A desktop computer (Computer 1) was used to build and present the VE for this study using FPS Creator. This software creates environments that are similar to a first-person shooter video game, in which a player sees everything from a viewer-based perspective. Figure 1 presents a view of part of a path that participants would traverse.

Participants indicated their estimates of path distance using E-Prime (version 1.1) on a second computer screen (Computer 2; $640 \times 480$ pixels). They were asked to draw the length of a specified path (using arrow keys) by comparing it with the length shown on the screen ( 80 pixels) for the comparison path (which was always the last path traversed and which had no turns). This comparative method is similar to the pencil-and-paper responses used in previous tests of the route angularity effect (Jansen-Osmann \& Berendt, 2002; Jansen-Osmann \& Wiedenbauer, 2004, 2006) and has been shown to produce results similar to other methods of distance estimation. We required participants to draw their estimates on a computer rather than on paper to increase our accuracy in collecting responses.

There were six path sets altogether, with each set containing three paths, similar to previous studies. The first two paths varied in length $($ small $=40 \mathrm{ft}$, medium $=80 \mathrm{ft}$, and large $=120 \mathrm{ft})$ and in the number of turns (two and seven). The last path was always $20 \mathrm{ft}$ in length, with no turns. The path lengths and the numbers of turns for each set are presented in Table 1. The corridors of the first path were always colored red, those of the second path were colored blue, and those of the third (comparison) path (with no turns) were colored yellow. ${ }^{1}$ Figure 2 provides a map of a representative path sequence. In addition to the mouse, which was used to navigate paths and to make estimates, a standard numeric keypad with all but four keys removed was used. Each of the four keys was given a new label-specifically, the 8 key was labeled "up," the 2 key "down," the 4 key "left," and the 6 key "right."

\section{Procedure}

Participants were given several practice trials to help them learn the mouse controls. They were also shown an example of the procedure, which included the navigation of a path with red walls, a path with blue walls, and then a path with yellow walls. They were instructed how to point the mouse cursor to adjust the length of a red

Table 1

Disruption Condition, Path Distances, and Number of Turns for the Six Experimental Path Sets

\begin{tabular}{ccccc}
\hline & & \multicolumn{3}{c}{ Distance (ft) } \\
\cline { 3 - 5 } $\begin{array}{c}\text { Path } \\
\text { Set }\end{array}$ & $\begin{array}{c}\text { Disruption } \\
\text { Task? }\end{array}$ & $\begin{array}{c}\text { First Path } \\
\text { (Two Turns) }\end{array}$ & $\begin{array}{c}\text { Second Path } \\
\text { (Seven Turns) }\end{array}$ & $\begin{array}{c}\text { Third Path } \\
\text { (Zero Turns) }\end{array}$ \\
\hline 1 & Yes & 40 & 40 & 20 \\
2 & No & 40 & 40 & 20 \\
3 & Yes & 80 & 80 & 20 \\
4 & No & 80 & 80 & 20 \\
5 & Yes & 120 & 120 & 20 \\
6 & No & 120 & 120 & 20 \\
\hline
\end{tabular}

Note-Path sets were presented in random order to the participants.

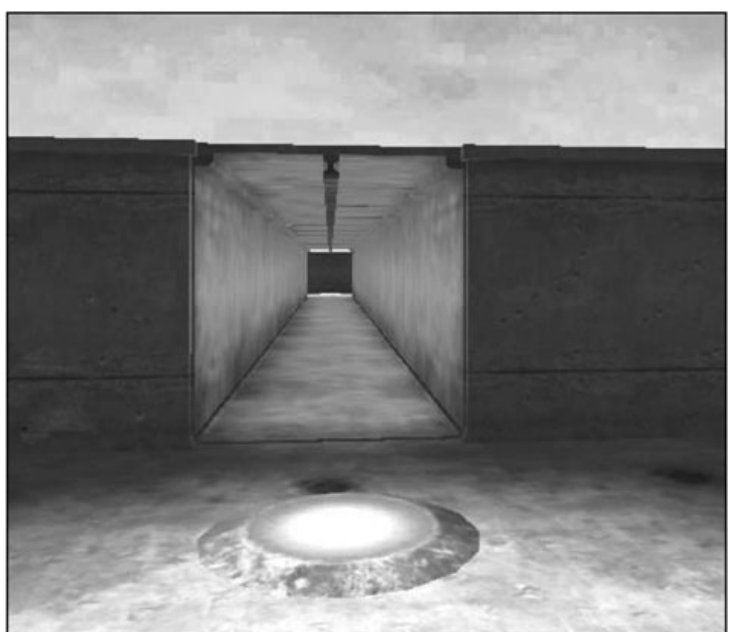

Figure 1. View of a part of a virtual path that the participants traversed. The lighted area on the floor in the initial chamber represented the starting point; a similar light represented the end point for a given path.

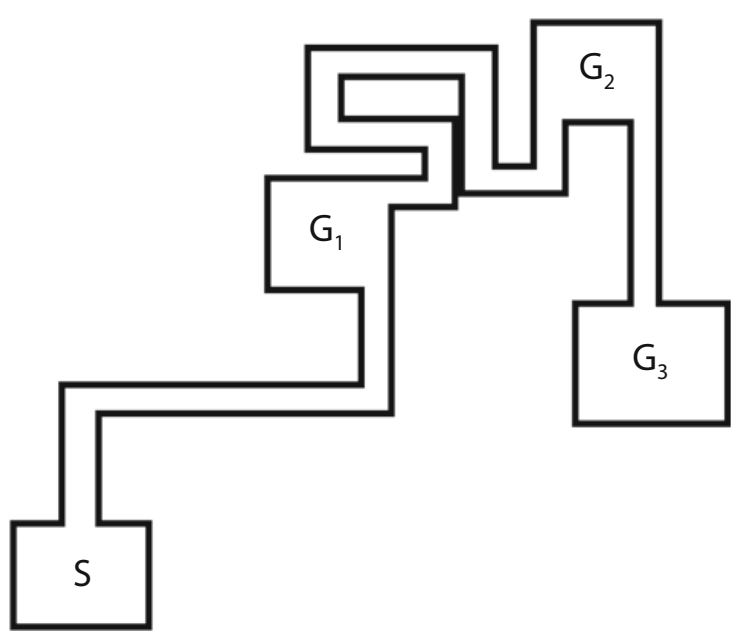

Figure 2. Example layout for paths. S, the start of the path set; $G_{1}$, the end of Path 1 and the starting place for Path $2 ; G_{2}$, the end of Path 2 and the starting place for Path 3; $G_{3}$, the end of Path 3.

line that represented the red path on the response screen to indicate its size relative to a yellow line ( 80 pixels in length) that represented the comparison path. They then did the same for a blue line that represented the blue path length.

The participants were then familiarized with the procedures involved in the concurrent task. They heard a series of vocalized directions from Computer 2 ("Up," "Down," "Left," and "Right") presented at a rate of one every $2 \mathrm{sec}$. The participants were required to use their left hand to press the arrow keys on the keypad each time they heard one of these spoken directions. During experimental trials, they did this direction-coding task while navigating the path. Prior to test trials, the participants were also familiarized with the Shepard-Metzler task described below.

The test trials consisted of six path sets. The timeline of a trial is shown in Figure 3. On relevant trials, the participants began the 


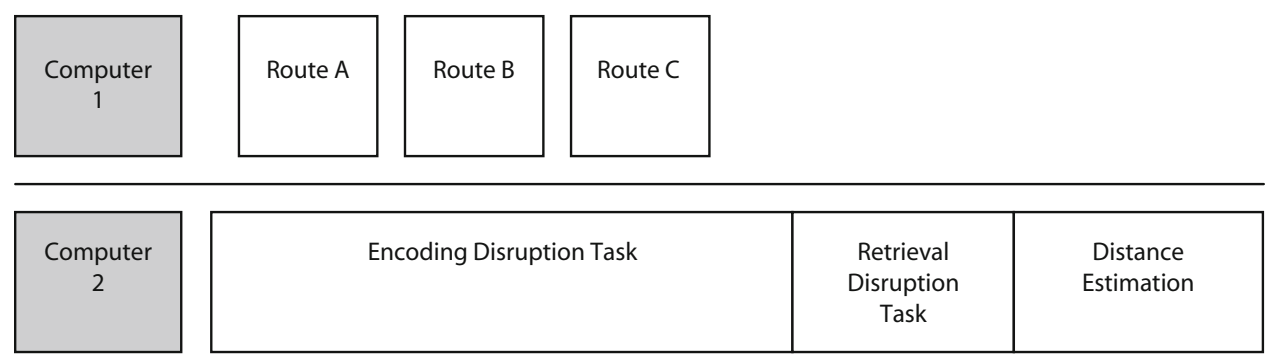

Figure 3. Timeline for a trial. Computer 1 was used to navigate through the virtual environment. Computer 2 was used to present the disruption tasks and to record all responses.

concurrent-direction coding task on Computer 2 before starting to navigate the paths. They then began theirVE navigation at the starting point room and moved down the red corridor, ending at the goal light on the floor of another chamber (see Figure 2). They then proceeded down blue and yellow paths as they continued the concurrent task. On the three trial sets for which there was no concurrent directioncoding task, they simply navigated the corridors. On reaching the end goal for the yellow path, they experienced a 60 -sec delay before estimating path lengths. In the filled-delay condition, participants were asked to complete a series of Shepard-Metzler mental rotation tasks in which a three-dimensional shape appeared on the top half of the screen, and two rotated shapes appeared on the bottom half (one being the rotation of the mirror image of the figure on the top half of the screen). The participants used the mouse to indicate which of the two figures was a rotated version of the top one and then received feedback for each judgment. This task was designed to occupy spatial working memory resources and so to disrupt mental rehearsal of the paths. The participants in the unfilled-delay condition simply waited for $60 \mathrm{sec}$ with no task to complete.

After the 60-sec delay, the participants looked at Computer 2, which showed two equal line lengths ( 80 pixels long) for red and yellow paths, with the yellow line below the red. They used the mouse to increase or decrease the length of the red line by clicking on the corresponding arrows on the screen until the relative lengths of the lines were the same as those of the paths. After indicating their responses, they estimated the relative size of the blue path in the same way. This procedure was repeated for each of the six path sets.

\section{RESULTS}

Given the much greater memory demands of these tasks, we expected that some of the participants might confuse paths and provide poor overall estimates of path lengths. Because including these participants might obscure the results, we eliminated data from 7 participants $(2$ men, 5 women) whose correlation between their estimated distances and the actual distances for the 12 estimates stood out as being quite poor $(r<.40)$. The average correlation for the remaining participants in this study was reasonably $\operatorname{high}(r=.77) .^{2}$

A $2 \times 2 \times 3 \times 2 \times 2$ ANOVA was performed on the distance estimates, with two between-subjects variables (sex of the participant and type of delay) and three withinsubjects variables (path size, number of turns, and concurrent task). (Figure 4 presents the path estimates as a function of path length and number of turns.) The results indicate that paths with more turns were estimated to be longer (the route angularity effect), with this effect weak- est for the longest path. Also, distance estimates were increasingly compressed as path size increased. This observation was confirmed by the ANOVA. The main effect of the path size $\left[F(2,58)=151.08, p<.001, \eta^{2}=.839\right]$ confirmed that the different path lengths were easily discriminated. The significant quadratic component of this effect reflected the greater compression of distance estimates with increased path lengths, with means for short, medium, and long paths being $M=44.83(S D=10.88)$, $M=62.80(S D=13.95)$, and $M=85.21(S D=16.22)$, respectively (actual lengths were 40, 80, and $120 \mathrm{ft}$ ).

The results also demonstrated a very strong route angularity effect, as was revealed in the significant main effect of the number of turns $[F(1,29)=50.52, p<.001$, $\left.\eta^{2}=.635\right]$. As is shown in Figure 4, distance estimates for paths with seven turns $(M=68.18, S D=14.00)$ were much greater than those for the paths with two turns $(M=$ $60.37, S D=9.76$ ). The effect size in this experiment was nearly three times greater than that reported by JansenOsmann and Wiedenbauer (2006) in their five-path study. Given the similarities in methodology across these studies, the enhanced route angularity effect may well be due to the increased memory demands.

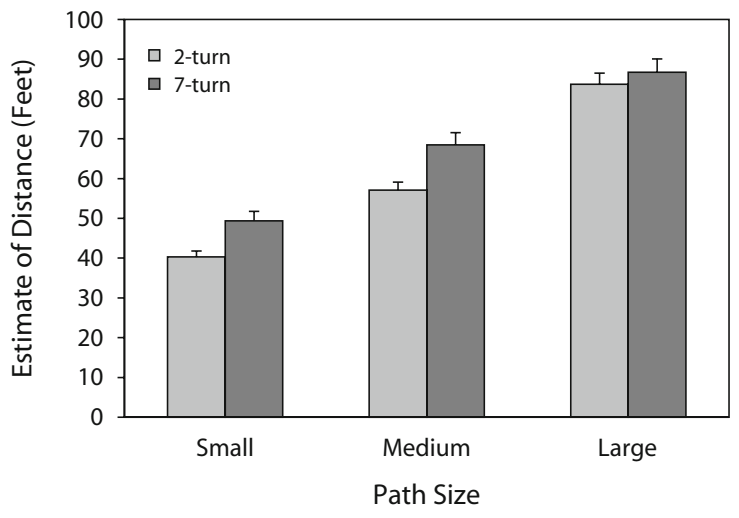

Figure 4. Estimates of distance as a function of path size and number of turns. The route angularity effect is reflected in the greater estimates for seven-turn paths. The actual path lengths were 40, 80, and $120 \mathrm{ft}$. Error bars represent 1 standard error of the mean. 
A

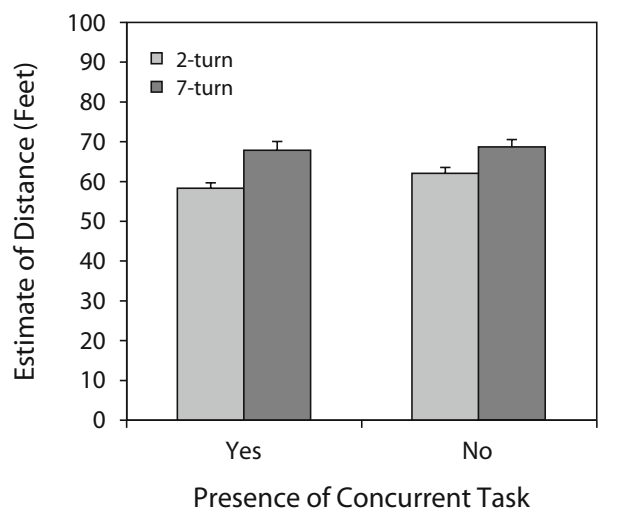

B

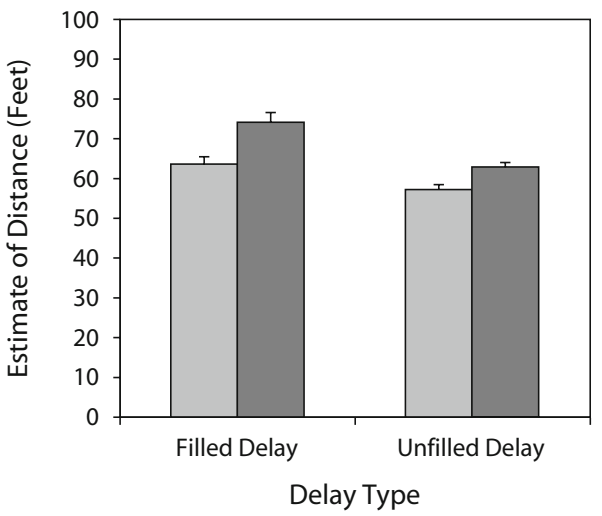

Figure 5. (A) Estimates of distance as a function of concurrent task and number of turns; the route angularity effect was greater for trials with the concurrent direction coding task than for trials without it. (B) Estimates of distance as a function of delay type and number of turns; the route angularity effect was greater in the filled-delay condition than in the unfilled-delay condition.

Consistent with the predictions of the dual coding model, there was a significant concurrent task $\times$ number of turns interaction $\left[F(1,29)=4.39, p<.05, \eta^{2}=.131\right]$. Figure $5 \mathrm{~A}$ shows that the magnitude of the route angularity effect was larger when the participant was concurrently engaged in the direction coding task (a mean difference of 9.38) than when there was no concurrent task (a mean difference of 6.24). The dual coding model also predicts a delay type $\times$ number of turns interaction, which was significant here $\left[F(1,29)=4.86, p<.05, \eta^{2}=.144\right]$. Figure 5B shows that the magnitude of the route angularity effect was larger when the participants completed the Shepard-Metzler task before giving an estimate (a mean difference of 10.53) than when the delay was unfilled (a mean difference of 5.55). The three-way interaction of number of turns with concurrent task and delay type was not significant $[F(1,29)=.086, p>.05]$, supporting independent influences of these two manipulations on the route angularity effect.

Note that there was a significant main effect of delay type $\left[F(1,29)=4.98, p<.05, \eta^{2}=.147\right]$, indicating that a filled delay increased estimates of distance $(M=69.15$, $S D=13.36)$ relative to an unfilled delay $(M=60.22$, $S D=8.26$ ). This result suggests that memory retrieval difficulties play some role in the estimation of distance. Furthermore, there was a significant interaction of concurrent task and path size $\left[F(2,58)=4.12, p<.05, \eta^{2}=\right.$ .124], reflecting poorer discrimination of distances when the participants conducted a concurrent spatial task. ${ }^{3}$

\section{DISCUSSION}

Our goal in this experiment was to directly test the memory basis of the route angularity effect. We did this by manipulating whether participants engaged in a concurrent spatial task during navigation and also by manipulating whether the delay before estimating path sizes was filled with a task that required spatial resources. Both of these tasks were designed to disrupt fine-grained memory for path distances and thus increase reliance on categorical memory, which would include information about the number of turns on each path. Our results were consistent with the prediction from the dual coding hypothesis that route angularity effects would increase when fine-grained memory representation is disrupted.

The significant interaction of concurrent task and number of turns provided evidence that disrupting encoding by increasing memory load during navigation enhances the route angularity effect. The significant interaction of delay type and number of turns provided evidence that taxing memory after the paths have been encoded through a spatial task that prevents rehearsal also leads to an enhanced route angularity effect. These effects appear to operate in an additive fashion. Taken together, we believe that a plausible memory-based explanation of this pattern of effects is that the number of turns is categorically encoded and used as a distance-estimation heuristic whenever analog or fine-grained memory for a path distance is disrupted. This account explains why disruption either at encoding or after encoding leads to a similar enhancement of the route angularity effect. It can also account for the findings of Sadalla and colleagues (Sadalla et al., 1979; Staplin \& Sadalla, 1981) that enhancing information about intersections and cues along the path increases the overestimation of distance. The dual memory explanation is that these manipulations enriched categorical memory, so that it was through this route that the biasing effects were achieved.

The present experiment extends our understanding of the route angularity effect, especially in VEs. In particular, we felt that there was a need to clarify and test the mechanisms guiding the route angularity effect in VEs. With an increasing amount of spatial research conducted in VEs as a replacement for real environments, it is neces- 
sary to better understand whether and how effects found in real-world spatial navigation extend to the virtual world. In addition, the rigorous controls available in VEs enable more flexible tests of cognitive mechanisms for processing spatial information.

Our experiment verified that the route angularity effect can be found in VEs, despite the rather capricious nature of this effect reported in the literature (Jansen-Osmann \& Berendt, 2002; Jansen-Osmann \& Wiedenbauer, 2004, 2006). However, our experiment went beyond the previous research by directly testing different memory accounts of the effect. This experiment provided direct evidence in favor of a memory basis of the route angularity effect. Although some researchers have speculated that the effect is based on increased memory load during navigation (Sadalla \& Magel, 1980), our results support a broader mechanism. This is because disrupting encoding during navigation had similar effects to disrupting rehearsal after navigation. These results are consistent with the idea that people tend to rely increasingly on a heuristic that uses the number of turns to estimate distance traveled along a path as memory demands increase. This mechanism is consistent with the general dual memory representation framework of the category adjustment model (Huttenlocher et al., 1991), which assumes that spatial information is coded at a fragile but highly accurate fine-grained level and a more robust but coarser categorical level. Memory demands, either at encoding or prior to retrieval, disrupt fine-grained memory more than categorical memory, and therefore estimates will increasingly rely on categorical memory, which, in this case, is based on the number of turns in the path.

Our results lead us to question the generality of the route angularity effect to less memory-demanding situations. The pattern of results reported by Jansen-Osmann and colleagues across a series of experiments (JansenOsmann \& Berendt, 2002; Jansen-Osmann \& Wiedenbauer, 2004, 2006) basically supports the assertion that reduction of memory demands results in a reduction or elimination of the route angularity effect. These results raise the question of whether the route angularity effect would be found in a study in which estimates are made after each path is navigated. We believe that the answer depends on the memory demands imposed by the task. For example, if the participants were concurrently processing other spatial information during navigation, the effect might well emerge. Such manipulations would provide a further test of the generality of the route angularity effect and its reliance on memory factors.

A factor that may be addressed in future studies is the visibility of the path itself. On the basis of Heft's (1988) analysis, the ability to see through some turns leads to a decreased route angularity effect in real-world settings. In our study, the seven-turn, $40-\mathrm{ft}$ path did include portions in which the participant could see in a straight line through a few of the turns. This was impossible to avoid given the length of the path and the number of turns that were required on the basis of previous experiments. If Heft is correct, then we should have found a reduced effect of angularity for those paths. However, the route angularity effect was large for our seven-turn $40-\mathrm{ft}$ paths relative to that for the two-turn 40-ft paths and was comparable to the effect for 80 -ft paths (see Figure 4). Thus, at least for novel VE paths, the ability of the viewer to see through the turns on a path did not seem to matter.

Another factor that we did not directly address in this research concerns how the route angularity effect may depend on intentions during learning. Some researchers have speculated that people will not rely on heuristics if they can acquire distance knowledge intentionally (JansenOsmann \& Wiedenbauer, 2006; Montello, 1997). For the present experiments, participants knew that they would be repeatedly estimating the distances of many paths before the experimental trials began. Clearly, we were able to find the route angularity effect even when intentional learning took place. Thus, our results imply that the critical issue is not intentionality of learning but, rather, the memory factors relating to the task. Naturally, if learning is incidental, memory may be poorer and may therefore lead to greater heuristic processing. Thus, the memory perspective can potentially account for results related to manipulating intentionality during learning.

\section{AUTHOR NOTE}

This experiment was conducted as part of the first author's dissertation. The authors dedicate this research to the memory of Gary L. Allen, who inspired it. Correspondence regarding this article should be addressed to A. T. Hutcheson (e-mail: hutchesa@mailbox.sc.edu).

\section{REFERENCES}

Allen, G. L. (1981). A developmental perspective on the effects of "subdividing" macrospatial experience. Journal of Experimental Psychology: Human Learning \& Memory, 7, 120-132. doi:10.1037/0278 $-7393.7 .2 .120$

Allen, G. L., \& Kirasic, K. C. (1985). Effects of the cognitive organization of route knowledge on judgments of macrospatial distance. Memory \& Cognition, 13, 218-227.

Briggs, R. (1973). Urban cognitive distance. In R. Downs \& D. Stea (Eds.), Image and environment: Cognitive mapping and spatial behavior (pp. 361-388). New Brunswick, NJ: Aldine.

Fitting, S., Wedell, D. H., \& Allen, G. L. (2008a). Cue usage in memory for location when orientation is fixed. Memory \& Cognition, 36, 1196-1216.

FitTing, S., Wedell, D. H., \& Allen, G. L. (2008b). External cue effects on memory for spatial location within a rotated task field. Spatial Cognition \& Computation, 8, 219-251.

Heft, H. (1988). The vicissitudes of ecological phenomena in environment-behavior research: On the failure to replicate the "angularity effect." Environment \& Behavior, 20, 92-99.

Huttenlocher, J., Hedges, L. V., \& Duncan, S. (1991). Categories and particulars: Prototype effects in estimating spatial location. Psychological Review, 98, 352-376. doi:10.1037/0033-295X.98.3.352

Jansen-OSMANN, P., \& BerEndt, B. (2002). Investigating distance knowledge using virtual environments. Environment \& Behavior, 34, 178-193. doi:10.1177/0013916502034002002

Jansen-Osmann, P., \& Wiedenbauer, G. (2004). The influence of turns on distance cognition: New experimental approaches to clarify the route-angularity effect. Environment \& Behavior, 36, 790-813. doi:10.1177/0013916503262537

Jansen-Osmann, P., \& Wiedenbauer, G. (2006). Distance cognition in virtual environmental space: Further investigations to clarify the routeangularity effect. Psychological Research, 70, 43-51. doi:10.1007/ s00426-004-0183-8

LeE, T. R. (1973). Psychology and living space. In R. M. Downs \& D. Stea 
(Eds.), Image and environment: Cognitive mapping and spatial behavior (pp. 87-108). New Brunswick, NJ: Aldine.

Merchant, H., Fortes, A. F., \& Georgopoulos, A. P. (2004). Shortterm memory effects on the representation of two-dimensional space in the rhesus monkey. Animal Cognition, 7, 133-143. doi:10.1007/ s10071-003-0201-z

Montello, D. R. (1997). The perception and cognition of environmental distance: Direct sources of information. In S. C. Hirtle \& A. U. Frank (Eds.), Spatial information theory: A theoretical basis for GIS (pp. 297-311). Berlin: Springer.

Sadalla, E. K., \& Magel, S. G. (1980). The perception of traversed distance. Environment \& Behavior, 12, 65-79. doi:10 $.1177 / 0013916580121005$

Sadalla, E. K., Staplin, L. J., \& Burroughs, W. J. (1979). Retrieval processes in distance cognition. Memory \& Cognition, 7, 291-296.

Shepard, R. N., \& MetZler, J. (1971). Mental rotation of threedimensional objects. Science, 171, 701-703. doi:10.1126/science .171 .3972 .701

Spencer, J. P., \& Hund, A. A. (2002). Prototypes and particulars: Geometric and experience-dependent spatial categories. Journal of Experimental Psychology: General, 131, 16-37. doi:10.1037/0096 $-3445.131 .1 .16$

Staplin, L. J., \& Sadalla, E. K. (1981). Distance cognition in urban environments. Professional Geographer, 33, 302-310.

STEA, D. (1969). The measurement of mental maps: An experimental model for studying conceptual spaces. In K. R. Cox \& R. G. Gollege (Eds.), Behavioral problems in geography: A symposium (pp. 228253). Evanston, IL: Northwestern University Press.

\section{NOTES}

1. Previous research has provided mixed results on whether the order of paths moderates the route angularity effect. One study showed an order effect (Jansen-Osmann \& Wiedenbauer, 2004), but most studies have shown no order effects (Jansen-Osmann \& Berendt, 2002; JansenOsmann \& Wiedenbauer, 2006; Sadalla \& Magel, 1980). In our experiment, the two-turn path always appeared first, and the seven-turn path always appeared second, so that the effects of order on the route angularity effect could not be assessed. Our focus was on the moderating effects of our memory manipulations on the observed effect. Since order was held constant, the memory manipulations were not confounded with order. Thus, although it would have been desirable to counterbalance the order of turns across sets, we believe that the effects of the memory manipulations that we report in this article can be unambiguously interpreted.

2. The results of an ANOVA conducted on the full set of participants provided the same pattern of significance for key effects presented in the Results section. Excluding those participants increased the effect size of most of the main effects and interactions reported.

3. The five-way design of Experiment 2 had 31 effects to test, and of these effects, 5 may be considered the focus of the experimental hypotheses. These effects were tested at an alpha of .05. To control Type I errors, the remaining 26 were tested using an alpha of .01, and none were found to be significant.

(Manuscript received March 6, 2008; revision accepted for publication December 17, 2008.) 